\title{
Does Right Ventricular Dysfunction Predict Mortality in Hemodynamically Stable Patients With Acute Pulmonary Embolism?
}

\author{
Ahmad Chaudhary, b, Umair Iqbal ${ }^{\mathrm{a}, \mathrm{b}, \mathrm{c}}$, Ayesha Jameel ${ }^{\mathrm{a}}$, Hafsa Anwar ${ }^{\mathrm{a}}$, \\ Edward Bischof ${ }^{\mathrm{a}}$
}

\begin{abstract}
Background: Acute pulmonary embolism (APE) is directly responsible for 100,000 deaths annually. Right ventricular dysfunction (RVD) on admission is considered a poor prognostic factor in these patients, though existing evidence of its significance in predicting mortality in hemodynamically stable patients is still unclear. We attempted to clarify this association by doing a retrospective review.
\end{abstract}

Methods: We retrospectively reviewed electronic medical records of hemodynamically stable patients older than 18 years of age with APE who were admitted to a tertiary care hospital in rural Upstate New York from July 2014 to July 2016. One hundred thirty-four patients were reviewed in two groups: patients who presented with computed tomography (CT) or echocardiographic evidence of RVD, and those without RVD. To identify differences in mortality between the two groups, the Chi-square/Fisher's exact test and $t$-tests were used. All variables with $\mathrm{P}<0.2$ in the initial analysis were included in a stepwise multivariable logistic regression model to predict RVD.

Results: No statistically significant difference was found in 30-day mortality between the groups (7.8\% in RVD and 5.3\% in no RVD, $\mathrm{P}=0.563$ ). The overall prevalence of RVD was found to be $57 \%$ (77/134). Troponin elevation (53.2\% in RVD group vs. 19.3 in the no RVD group with $\mathrm{P}<0.01)$ and central location of thrombus $(53.1 \%$ vs. $32.1 \%$ with $\mathrm{P}=0.016)$ were more prevalent in RVD group. A marginally significant difference was found in length of hospital stay among those with RVD versus no RVD (7.13 days vs. 5.46 days; $\mathrm{P}=$ 0.061). The multivariable analysis shows that the odds of RVD were greater for patients with elevated troponin levels (odds ratio $=7.8$ ).

Conclusion: There was no difference in 30-day mortality in hemodynamically stable patients with APE having RVD compared to patients with no RVD. On the basis of this study, we do not suggest the routine

Manuscript submitted July 19, 2017, accepted August 1, 2017

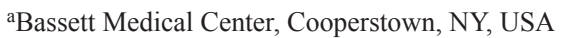

${ }^{b}$ These authors contributed equally and shared the first authorship.

${ }^{\mathrm{c} C o r r e s p o n d i n g ~ A u t h o r: ~ U m a i r ~ I q b a l, ~ B a s s e t t ~ M e d i c a l ~ C e n t e r, ~ O n e ~ A t w e l l ~}$

Road, Cooperstown, NY 13326, USA. Email: umair.iqbal@bassett.org

doi: https://doi.org/10.14740/cr577w use of systemic fibrinolysis in hemodynamically stable patients with radiographic evidence of RVD alone.

Keywords: Right ventricular dysfunction; Acute pulmonary embolism; Fibrinolysis

\section{Introduction}

Acute pulmonary embolism (APE) is directly responsible for 100,000 deaths annually in United States [1]. It is associated with high inpatient mortality. APE can be classified as massive or submassive. Patients with massive PE present with hemodynamic instability and are usually treated with systemic thrombolysis or embolectomy [2-4]. Patients with submassive $\mathrm{PE}$ are hemodynamically stable on presentation but have either elevated cardiac biomarkers (brain natriuretic peptide (BNP) or troponin) or echocardiographic evidence of right ventricular dysfunction (RVD). Many studies show RVD to be associated with higher short-term mortality [5-9]. Though these patients are usually treated with anticoagulation alone, guidelines suggest considering treatment of some high-risk patients with submassive PE with evidence of RVD with systemic fibrinolysis $[10,11]$. Some studies also show no difference in mortality and recommend against using any systemic thrombolysis in these hemodynamically stable patients [12].

Therefore, existing evidence of RVD significance in predicting mortality in hemodynamically stable patients is still unclear. We conducted a retrospective study to clarify if RVD on computed tomography (CT) or echocardiogram in hemodynamically stable patients with APE is associated with increased mortality.

\section{Patients and Methods}

We retrospectively reviewed electronic medical records of hemodynamically stable patients older than 18 years of age with APE who were admitted to a tertiary care hospital in rural Upstate New York from July 2014 to July 2016. All patients over 18 years of age admitted with diagnosis of APE (ICD 10 codes: I26.0, I26.9) were included in the study. Patients who 
Table 1. Association of RVD With Other Characteristics

\begin{tabular}{|c|c|c|c|}
\hline \multirow{2}{*}{ Characteristics } & \multicolumn{2}{|c|}{ RVD } & \multirow{2}{*}{ P-value } \\
\hline & No $(n=57)$ & Yes $(n=77)$ & \\
\hline Age (mean, SD) & $62.9(16.9)$ & $64.5(15.0)$ & 0.54 \\
\hline Male & $30(52.6)$ & $40(51.9)$ & 0.938 \\
\hline Female & $27(47.4)$ & $37(48.1)$ & \\
\hline Obesity & $24(42.1)$ & $46(59.7)$ & $0.043 *$ \\
\hline History of COPD & $10(17.5)$ & $9(11.7)$ & 0.337 \\
\hline History of CAD & $11(19.3)$ & $11(14.3)$ & 0.439 \\
\hline History of CHF & $9(15.8)$ & $11(14.3)$ & 0.809 \\
\hline Diabetes & $13(22.8)$ & $19(24.7)$ & 0.802 \\
\hline Chronic kidney disease & $1(1.8)$ & $9(11.7)$ & $0.031 *$ \\
\hline Smoker & $30(52.6)$ & $38(49.4)$ & 0.707 \\
\hline Malignancy & $18(31.6)$ & $24(31.2)$ & 0.960 \\
\hline Recent surgery & $8(14)$ & $12(15.6)$ & 0.803 \\
\hline Immobility & $13(22.8)$ & $20(26)$ & 0.674 \\
\hline Calf tenderness & $7(12.3)$ & $11(14.3)$ & 0.736 \\
\hline Leg swelling & $11(19.3)$ & $11(14.3)$ & 0.439 \\
\hline Hemoptysis & $4(7)$ & $1(1.3)$ & 0.084 \\
\hline Shortness of breath & $41(71.9)$ & $64(83.1)$ & 0.120 \\
\hline Syncope & $4(7)$ & $5(6.5)$ & 0.905 \\
\hline Chest pain & $29(50.9)$ & $27(35.1)$ & 0.067 \\
\hline Troponin & $11(19.3)$ & $41(53.2)$ & $<0.01^{*}$ \\
\hline D-dimer & $27(64.3)$ & $36(60)$ & 0.661 \\
\hline History of DVT/PE & $18(31.6)$ & $17(22.1)$ & 0.216 \\
\hline Death in 30 days & $3(5.3)$ & $6(7.8)$ & 0.563 \\
\hline Central location of thrombus & $18(32.1)$ & $39(53.1)$ & $0.016^{*}$ \\
\hline
\end{tabular}

${ }^{*} \mathrm{P}<0.05$ was considered significant using Pearson Chi-square test.

developed APE during hospitalization were excluded from the study. APE was diagnosed on the basis of CT scan, high probability V/Q scan or venous Doppler ultrasound with associated symptoms. One hundred thirty-four patients met the inclusion criteria and were reviewed in two groups: patients who presented with CT or echocardiographic evidence of RVD, and those without RVD.

We defined RVD as RV/LV ratio $>0.9$ or RV hypokinesis on echocardiogram as defined by the American Heart Association or RV dilation on CT scan. Location of thrombus was classified as central if it was located in one of the main pulmonary arteries or peripheral if involving segmental, sub-segmental or lobar arteries. Hemodynamic instability was defined as systolic blood pressure (BP) of $<90 \mathrm{~mm} \mathrm{Hg}$ on initial presentation. Patients with BP of $<90 \mathrm{~mm} \mathrm{Hg}$ were excluded from the study.

\section{Statistical analysis}

To identify univariate associations between RVD and subject characteristics, the Chi-square/Fisher's exact test and $t$-tests were used (Table 1). P value of $<0.05$ was considered statistically significant. All variables with $\mathrm{P}<0.2$ in the initial analysis were included in a stepwise multivariable logistic regression model to identify independent predictors of RVD (Table 2). All analyses were carried out using SPSS 23.0 version.

\section{Results}

The prevalence of RVD was found to be $57 \%$ (77/134). Central localization of the clot was present in $42 \%$ of patients $(57 / 134)$. Shortness of breath was the most common presenting symptom followed by chest pain. On physical exam, calf tenderness and leg swelling were common. Troponin elevation, defined as troponin $>0.05$, was present in $39 \%(52 / 134)$ of patients.

The overall 30-day mortality associated with APE was $6.7 \%(9 / 134)$. No statistically significant difference was found in 30-day mortality between patients with RVD vs. no RVD (7.8\% vs. $5.3 \%$; confidence interval $(\mathrm{CI})=0.68-2.34 ; \mathrm{P}=$ 0.563 ). Marginally significant difference was found in length of stay in patients who had evidence of RVD (mean 7.13 days 
Table 2. Odds Ratio and $95 \%$ Confidence Interval for RVD

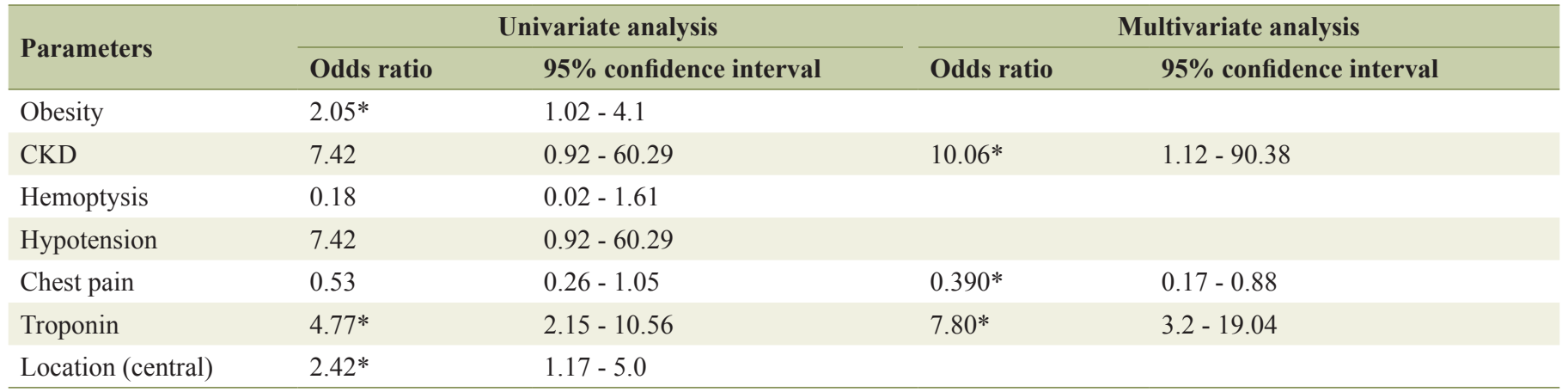

${ }^{*} \mathrm{P}<0.05$ was considered significant.

in patients with RVD vs. 5.46 without RVD, $\mathrm{P}=0.061)$. Central localization $(53.1 \%$ with RVD vs. $32.1 \%$ without RVD, P $=0.016)$ and troponin elevation $(53.2 \%$ in patients with RVD vs. $19.3 \%$ without RVD, $\mathrm{P} \leq 0.001)$ were found to be more prevalent in RVD patients.

Chronic kidney disease as defined by glomerular filtration rate $(\mathrm{GFR})<60 \%$ and obesity defined by body mass index (BMI) of $>35$ were found to be more prevalent in the RVD group. No difference was found in other comorbidities in the two groups including coronary artery disease (CAD), diabetes, chronic obstructive pulmonary disease (COPD) and congestive heart failure (CHF).

All variables with $\mathrm{P}<0.2$ were included in a stepwise multivariable logistic regression model to predict RVD (Table 2). Troponin elevation (odds ratio $(\mathrm{OR})=7.8, \mathrm{CI}=3.2$ - 19.04) and chronic kidney disease $(\mathrm{CKD})(\mathrm{OR}=10.06, \mathrm{CI}=1.12$ 90.38) were significantly higher among those with RVD. Troponin was retained as an independent predictor of RVD after stepwise selection.

\section{Discussion}

Systemic hypotension and shock in the presence of evidence of acute right heart dysfunction are major predictors of poor outcomes in APE and are the indications for the use of systemic fibrinolytic or embolectomy [2-4]. The controversial scenario is the presence of right heart dysfunction in the presence of no hemodynamic instability. Current guidelines support the use of systemic thrombolysis in these hemodynamically stable patients with RVD $[10,11]$. Although the evidence for increased mortality in APE with RVD is well documented in the literature, its significance in normotensive patients in predicting mortality is still unclear.

The prevalence of RVD varies from $30 \%$ to $80 \%$ depending on the definition used [12]. The lack of standard criteria is the reason for the wide variability. In our study, we used the American Heart Association definition of RVD. A meta-analysis done on 3,395 patients admitted with PE revealed a twofold increase in mortality, but sub-analysis on normotensive patients correlated poorly with mortality [13]. Similar results were seen in a study done in 400 patients older than 65 years of age, which showed that RVD in APE was not associated with poor outcomes [12]. Our study supports these findings that normotensive patients with APE are not at increased risk for 30-day mortality, although hemodynamic instability with RVD is associated with increased mortality. Our study also does not support the American Heart Association recommendation of using fibrinolysis in hemodynamically stable patients with APE with radiographic evidence of RVD given no mortality difference.

There are several potential explanations for our results. First RVD may be a sign of other cardiopulmonary disease in normotensive patients and not related to APE [14, 15]. Second, in elderly populations, who comprised a large percentage of our study, mortality is more driven by multiple comorbidities rather than the acute episode.

In 2014, a randomized controlled trial (PEITHO) of normotensive patients with APE and radiographic evidence of RVD evaluated the difference in mortality between fibrinolysis with tenecteplase and placebo. Hemodynamic decompensation was reduced in the fibrinolysis group, but with an increased risk of bleeding complications. Most importantly there was no difference in all-cause mortality at 7 and 30 days between both the groups [16].

\section{Conclusion}

There was no difference in 30-day mortality in hemodynamically stable patients with APE having RVD compared to patients with no RVD. Troponin elevation was associated with significantly increased odds of RVD. The findings from this retrospective review do not provide evidence for aggressive management of hemodynamically stable patients with evidence of RVD with fibrinolytics or embolectomy as recommended in some prior studies. Physicians may want to consider restricting those treatment options to hemodynamically unstable patients with APE.

\section{Acknowledgments}

We acknowledge Bassett Research Institute, Bassett Medical Center (Cooperstown, NY), and Jennifer Victory, RN, from Bassett Medical Center. 


\section{References}

1. Horlander KT, Mannino DM, Leeper KV. Pulmonary embolism mortality in the United States, 1979-1998: an analysis using multiple-cause mortality data. Arch Intern Med. 2003;163(14):1711-1717.

2. Wan S, Quinlan DJ, Agnelli G, Eikelboom JW. Thrombolysis compared with heparin for the initial treatment of pulmonary embolism: a meta-analysis of the randomized controlled trials. Circulation. 2004;110(6):744-749.

3. Kearon C, Akl EA, Ornelas J, Blaivas A, Jimenez D, Bounameaux H, Huisman $\mathrm{M}$, et al. Antithrombotic Therapy for VTE disease: CHEST guideline and expert panel report. Chest. 2016;149(2):315-352.

4. Kearon C, Akl EA, Comerota AJ, Prandoni P, Bounameaux H, Goldhaber SZ, Nelson ME, et al. Antithrombotic therapy for VTE disease: Antithrombotic therapy and prevention of thrombosis, 9th ed: American college of chest physicians evidence-based clinical practice guidelines. Chest. 2012;141(2 Suppl):e419S-e496S.

5. Grifoni S, Olivotto I, Cecchini P, Pieralli F, Camaiti A, Santoro G, Conti A, et al. Short-term clinical outcome of patients with acute pulmonary embolism, normal blood pressure, and echocardiographic right ventricular dysfunction. Circulation. 2000;101(24):2817-2822.

6. Task Force on Pulmonary Embolism, European Society of Cardiology. Guidelines on diagnosis and management of acute pulmonary embolism. Eur Heart J. 2000;21(16):1301-1336.

7. Goldhaber SZ, Visani L, De Rosa M. Acute pulmonary embolism: clinical outcomes in the International Cooperative Pulmonary Embolism Registry (ICOPER). Lancet. 1999;353(9162):1386-1389.

8. Becattini C, Casazza F, Forgione C, Porro F, Fadin BM, Stucchi A, Lignani A, et al. Acute pulmonary embolism: external validation of an integrated risk stratification model. Chest. 2013;144(5):1539-1545.
9. Coutance G, Cauderlier E, Ehtisham J, Hamon M, Hamon $\mathrm{M}$. The prognostic value of markers of right ventricular dysfunction in pulmonary embolism: a meta-analysis. Crit Care. 2011;15(2):R103.

10. Jaff MR, McMurtry MS, Archer SL, Cushman M, Goldenberg N, Goldhaber SZ, Jenkins JS, et al. Management of massive and submassive pulmonary embolism, iliofemoral deep vein thrombosis, and chronic thromboembolic pulmonary hypertension: a scientific statement from the American Heart Association. Circulation. 2011;123(16):1788-1830.

11. Konstantinides SV, Torbicki A, Agnelli G, Danchin N, Fitzmaurice D, Galie N, Gibbs JS, et al. 2014 ESC guidelines on the diagnosis and management of acute pulmonary embolism. Eur Heart J. 2014;35(43):3033-3069, 3069a-3069k.

12. Hofmann E, Limacher A, Mean M, Kucher N, Righini M, Frauchiger B, Beer JH, et al. Echocardiography does not predict mortality in hemodynamically stable elderly patients with acute pulmonary embolism. Thromb Res. 2016;145:67-71.

13. ten Wolde M, Sohne M, Quak E, Mac Gillavry MR, Buller HR. Prognostic value of echocardiographically assessed right ventricular dysfunction in patients with pulmonary embolism. Arch Intern Med. 2004;164(15):1685-1689.

14. Wilson SR, Ghio S, Scelsi L, Horn EM. Pulmonary hypertension and right ventricular dysfunction in left heart disease (group 2 pulmonary hypertension). Prog Cardiovasc Dis. 2012;55(2):104-118.

15. Freixa X, Portillo K, Pare C, Garcia-Aymerich J, Gomez FP, Benet M, Roca J, et al. Echocardiographic abnormalities in patients with COPD at their first hospital admission. Eur Respir J. 2013;41(4):784-791.

16. Meyer G, Vicaut E, Danays T, Agnelli G, Becattini C, Beyer-Westendorf J, Bluhmki E, et al. Fibrinolysis for patients with intermediate-risk pulmonary embolism. N Engl J Med. 2014;370(15):1402-1411. 